\title{
Discussion on Opera Dance's Function in Opera Performance
}

\author{
Xuemei Chen \\ Music and Dance College \\ Qujing Normal University \\ Qujing, Yunnan, China 655011
}

\begin{abstract}
Nowadays Chinese economy is developing rapidly, and people's love and attention to traditional culture become increasingly prominent, which is shown in various artistic creation. Taking opera performance as an example, as a kind of comprehensive artistic performance form, its main content is to show people's abundant life and tortuous stories. To better show the infinite charm of opera performance, in recent years, opera dance gradually becomes an important part of opera performance, which can better show the opera actors' emotions in the performing process than pure opera performance. We can say that opera dance promotes our country's opera performance progress in some degree, better satisfies people's aesthetic psychology with the development of social tendency, and infuses a fresh vitality in our country's opera performance. Focusing on this phenomenon, the paper will make a simple discussion on the opera dance's function in opera performance.
\end{abstract}

Keywords-traditional culture; opera performance; opera dance; function; discussion

\section{INTRODUCTION}

Chinese dance culture has a long history, which is the wisdom crystallization of Chinese nation. When dance is combined with opera, what contained is more profound cultural artistic connotation. Along with the excellent performing skills of dance actors, people's production, life style, religious belief and folk customs reflected in opera performance are presented in front of the public more vividly, and virtually add the infection of opera performing art. Opera dance was born with the continuous progress and development of Chinese opera performance, and has essential status as an important part of opera performance and popular performing form. At the same time, it claims stricter requirement to the dancing basis of opera actors. This is undoubtedly a great impact and challenge to the development of present opera performance of our country.

\section{THE BIRTH PROCESS OF OPERA}

\section{A. The Implication of Opera}

The opera is a kind of traditional Chinese drama form with a long history. It is an independent artistic performance, but it contains various performance arts such as literature, music, dance, fine art, martial art and acrobatics. The opera has a more complete form and content after a long developing process, and forms it own special artistic performance form.

\section{B. Origin of Opera}

Chinese opera culture has a long history. A seemingly elegant artistic performance actually came from imitation of laborers' song and dance at the earliest. The opera first sprouted in pre-Qin period. In "Song" in "The Book of Songs" and "Nine songs" in "The Songs of Chu", it was only a libretto of songs and dances in sacrifice of immortals. Up to the Spring and Autumn Period and the Warring States Period and Han Dynasty, the opera began to evolved to song and dance entertaining human from that entertaining immortals, which was a great progress for operas at that time. Up to Tang Dynasty, the opera began to enter the forming period, and it was in Yuan Dynasty that it got really mature, actually, it also developed based on "Yuan poetic drama" and formed a kind of new drama after continuous development and evolution.

\section{Overview of Opera}

As far as art classification is concerned, the opera belongs to drama, actually a kind of drama, among which stage drama, pantomime, opera and dance drama are common. As a part of dramas, there are great differences among them. For example, opera and dance drama are combined with music, but stage opera is seldom combined with music or dance, which is a typical feature of the stage drama. With the development of society and change of people's concept, each of the drama performance will keep fusing with other performance features, which has special meaning to the future development of Chinese drama career.

\section{Various Expressing Method of Opera}

Singing, acting, recitation and acrobatics are four basic skills of actors in Chinese opera performance. When actors are performing opera, they need to clearly know how to make gestures, how to express their own emotions more completely with organs such as eyes. This is a relatively fixed model in Chinese opera performance. There might be many virtual actions in the whole performing process, but it is a true reality to actual life. Along with the beautiful dance of actors and seasonable music of the actors, opera performance will be further beautified and be more vivid. 


\section{FEATURE OF OPERA DANCE}

\section{A. The Prop Is Distinctive}

If the opera actors want to vividly show the vivid plot and complicated emotion in opera performance, there is great limit to merely depend on body movement and high skill. Thus, besides solid basic skills, dance actors also need to perform via prop related to opera them, such as fans which are very common in opera dance. No matter the opera actors take them in the hands or wear them on the head, or tie them on waist, there will be different effects. Fans are treated as a prop necessary for performance, but excellent opera dance actors can make them part of their own body. They can vividly show beautiful dancing posture of actors and various stories in the opera.

\section{B. Diversified Expressing Modes}

Our country's opera performance has been mature so far, but there is still certain disadvantage. Most often, the part which can not be shown by opera performance, especially the emotion of characters needs to be expressed via opera dance. The most common is to express the posture of birds and animals, whose movement needs to be well balanced and controlled. It's believed that only opera dance can express so fully.

\section{Strong Expressive Force}

As an opera dance actor, his every word and action have a basis, especially the use and selection of props shall better show the plot and even conflict in opera performance. For example, an ancient romantic young man took a fan in the hand not only for cooling, but to better express his emotion. Each action such as opening the fan, drooping the fan and folding the fan has a reason. The prop is combined with the dance performance of actors, which makes whole opera performance more expressive.

\section{Excellent Skill}

Opera dance is often expressed via the excellent acting skills of actors, thus the requirement of actors is particularly strict. Not only do they have to understand music, but also they shall be able to dance, which can be said as being good at singing and dancing. The requirement of actors is high because only solid opera performing basic can exert the creativity of actors themselves, and enable them to act freely in future opera performance. Opera dance is not the same as general folk dance or classical dance, and only after patient observation, research and study can actors show their excellent skills on the opera stage, and get accepted by more people. All these focus on silent persistence and continuous paying.

\section{PERFORMING SKILl OF OPERA DANCE}

\section{A. Excellent Dance Actors}

Opera dance is not the same as general modern dance, which has more strict requirements to the actors, thus actors shall do their best during performance. Firstly, the training of basic skills shall exercise waist skill, after all in opera performance, sometimes the dance needed to display is relatively complicated and diversified according to plot. Thus the training in peacetime shall be targeted. Of course, besides, waist, the hands and feet shall keep in high flexibility, if any of the dancing action is mechanical, not only does it make audience sick, but also the whole opera performance will fail. Besides, after opera dance actors practice basic skills, they shall also notice to keep beautiful dancing posture, at least combine each dancing action with plot in opera. If the dancing action can not express the theme of performance, it has no value of existence at all. Thus a good opera dance actor shall remember that the dance and acting supplement each other and can not be partitioned all the time.

\section{B. Fusion of Music Rhythm Sensation}

The rhythm is not only a part of music, but also one of the elements constituting dance actions. The actions of opera dance always combine activity and inertia, and the distinctive rhythm sensation is a most prominent feature of opera dance. How opera dance performs different rhythm sensation changes with the plot of the opera performance. As people can see, most opera dance is expressed via musical instruments like gong and drum, which is also an important mark of Chinese opera performance. Once the story plot presented on the stage progresses, the actors' movements shall rapidly speed up, and the emotion will also upsurge, the exciting sound of gong and drum implies that the actors need to speed up dancing steps, and the dancing movement will slow down a lot instead. Thus, the actors shall fully combine the detailed condition during performance to express in detail, grasp scale and pay attention to details, which is very important. Any artistic performing form actually comes from life, only it is higher than life after being processed into artistic expressing mode. If opera dance really wants to break through in opera performance, the actors shall go deep into life, carefully observe everything of life, and only so can roles shaped by actors be more vivid.

\section{True Reflection of Real Life}

Opera dance is different from other dancing arts, mainly because it is branded with the mark of opera, it also change with the change of opera performance. Besides being a kind of performing art, opera dance is also a kind of visual art. Audience can clearly see everything presented on the stage by the actors. Although there is not much dancing movement in some opera performances, it is the true reflection of real life, and such situations only arise after deep processing of actors.

\section{OPERA DANCE'S FUNCTION IN OPERA PERFORMANCE}

\section{A. Express Life Vividly}

The opera performance expresses via the form of dance, which is early dramatized, and opera dance has already been fused in all the movements and expressions of actors on the stage, from coming out to going off the stage, the gestures and expressions of the actor present excellent performing art. In fact, all the daily activities of people including basic necessities of life have come up on the stage of opera, only danced. On the stage of opera, once there is sound, there is song, once there is action, there is dance. It can be seen that 
dance is closely connected to opera, which is also the basic feature of present Chinese opera performance. In opera performance, a kind of life is shown via opera dance. Ultimately, dance and performance come from real life, only processed into a kind of new artistic expressing mode. It can be known that although opera dance and opera performance contain exaggerated expressing form, they are common life style.

\section{B. Express Emotions}

Opera dance can not only express the content of opera performance, but can also better express emotions. Some traditional Chinese classical dance focuses on emotion expression, mainly expressing the character's emotion via shape, movement of the actor to better shape the character's personality and express emotions. The opera dance is a kind of silent language, only depending on beautiful shapes of dance actors with strong function of emotion expression. The opera dance is silent, but it has a set of abundant dance performing form. For example, when the opera actor flips hair hard, he or she is expressing an excited emotion. These seemingly casual movements are actually a kind of method to express emotions and spirit, reaching the highest artistic realm of "silence better than sound".

\section{Infinite Charm of Artistic Creation}

The opera dance shapes artistic image of beauty via dancing language on the opera stage. The opera dance has various expressing methods and different functions. It can not spread sound, but it can reflect people's truest emotions via performing forms of singing, acting, recitation and acrobatics, and can even express good and evil, beauty and ugliness in real life via excellent artistic forms. Especially in character expression, it can even express pretty features. Even those cruel scenes of fighting would not make people frightened after processing of opera dance. Sometimes, being nervous and thrilled is an enjoyment of beauty. Opera dance can shape all the virtual or real images but will not limit the image, from which it can be seen that it has strong plasticity and wide adaptability.

\section{Reflection of Value of the Time}

The opera comes from life and is higher than life, so both opera dance and opera performance are actually closely connected to the time. Opera dance with connotation can shape different images and show the internal spiritual temperament, so the image will be more vivid on the stage and full of infinite charm and leave a deep impression on people.

\section{E. Inheritance of Culture}

As an old artistic expressing mode, opera has a long history in the history of Chinese opera. It not only contains profound artistic deposits, but also reveals people's daily life style, religious belief, folk custom, ethical ideas, moral rule and aesthetic taste, which not only offers audience wide vision, but also strengthens the infection of opera performing art, also a reflection of important artistic feature of opera performance. There has been great change for opera developing so far, but it is a continuity and inheritance to traditional Chinese opera culture, and combining dance to opera will enable Chinese opera to keep exerting its infinite vitality.

\section{Future DEVEloping TEND OF OPERA DANCE}

The formation and development of opera dance is a process of opera absorbing and fusing dance. Developing so far, opera dance not only includes abundant national cultural connotation, but also refers to and innovates large amounts of foreign cultures, and so forms its special advantage. The opera dance would not develop so far if there were no continuity and inheritance to traditional dance and absorption of traditional folk dance. Even though there is great development in opera dance as an individual artistic performing form today, its future developing tend shall also be paid attention to. It can be seen from current developing tend of opera that there is no competitive advantage for opera dance alone. To go on to carry forward dance in performance, it has to choose to combine opera and performance, and become the performance in opera, of course, it also needs to strengthen via dance for opera to go on.

\section{CONCLUSION}

Chinese opera performance has a long history, which is the essence and sediment of Chinese culture, also an enjoyment and pursuit of beauty after people's living standard improves. So its existing value shall not be underestimated. Only with the development and change of society, there is some bottleneck for Chinese opera performance. Seeking for solution focusing on this problem is essential now. After continuous exploration, it's found that fusing dance in opera performance is very natural, and practice proves that it injects new vitality to opera performance.

Opera art has a long influence on the field of Chinese art, especially it has a profound influence on creation of Chinese dance, in return, opera dance also promotes the rapid development of opera performance. At present, Chinese economy is developing fast, and even though people's material life is satisfied, the development of traditional culture shall not be ignored.

\section{REFERENCES}

[1] Zhang Limin, Opera and Dance. [M]. China Film Press. 2014.11

[2] Wang Wei, University students' artistic culture (appreciation of music, dance, opera and film) $[\mathrm{M}]$. University of Science \& Technology of China. 2009.07

[3] Huang Kebao, Research on opera performance [M]. Culture and Art Publishing House. 2014.01

[4] Jia Zhigang, Discussion on property of opera performance: unification between experience and expression. [M]. Beijing Shidaihuawen publishing house. 2015.03

[5] Ouyang Zhongshi, Research on Chinese opera performance system [M]. Culture and Art Publishing House. 2012.10 